\title{
Comparison of Smallpox vs. HIV/AIDS Eradication Strategies
}

Annise Chung and Murray R Berkowitz

Preventive and Community-Based Medicine, Department of Osteopathic Manipulative Medicine, Philadelphia College of Osteopathic Medicine, Georgia Campus, 625 Old Peachtree Road NW, Suwanee, Georgia 30024, USA

\begin{abstract}
In 2011, an estimated 34 million people globally were living with HIV, 2.5 million people became newly infected, and 1.7 million people died from AIDS-related illnesses. Although there has been a reduction of 700,000 new HIV cases since 2001, HIVIAIDS remains one of the leading causes of death globally. Since there is no known cure or vaccine currently present, preventing infection has become central to controlling the HIVIAIDS epidemic. With the United Nation's goal of "Getting to Zero" for the next generation, it may be warranted to review other successful global eradication strategies. The most well known global eradication may be the smallpox eradication program. Although both strategies aim to eradicate diseases, eradication of the HIVIAIDS epidemic has remained much more elusive. This paper will strive to demonstrate the similarities of the smallpox and HIVIAIDS global eradication programs, and the unique challenges HIVIAIDS poses for successful eradication.
\end{abstract}

\section{Introduction}

In 2011, the World Health Organization (WHO) estimated that 34 million people globally were living with HIV, 2.5 million people became newly infected, and 1.7 million people died from AIDS-related illnesses. [1] Although there has been a reduction of 700,000 new HIV cases since 2001 [1], HIV/AIDS remains one of the leading causes of death globally. Thus, the estimated prevalence of HIV is over 480 per 100,000 ; the estimated incidence is over 35 per 100,000. Since there is no known cure or vaccine, preventing infection has become central to controlling the HIV/AIDS epidemic.

In 1958, Professor Viktor Zhdanozv, Deputy Minister of Health of the USSR, presented a report arguing that the issue of smallpox was important to all nations, eradication was feasible and that national programs had demonstrated it to be practically possible. Subsequently, in 1959, the WHO decided to undertake the global eradication of smallpox. Thus began the development of the Global Smallpox Eradication Programme, which would end a little more than 18 years later with the successful eradication of smallpox [2].

As reported by Fenner and associates, political "buy in" or commitment was essential [2]. The original 1958 report had eradication efforts centralized on vaccination and re-vaccination campaigns. A subsequent program goal in 1959 proposed having $80 \%$ of the world population either vaccinated or revaccinated, with vaccination responsibility primarily dependent on the individual governments. Although no records of marking countries as endemic to smallpox existed at the time, it is surmised that in 1959, 59 countries and territories in Africa, South America, and Asia were endemic to smallpox. Due to these initial vaccination efforts, in 1967, it was estimated that only 31 countries or territories was endemic to smallpox, compared to the 59 countries and territories estimated in 1959. However, despite these efforts, smallpox still remained a large public health issue [2].

In 1966, an intensified eradication program was developed, catalyzed by 2 developments: the improvements of the jet injector and the commitment by the USA to provide support of the smallpox eradication program. The improvements of the jet injector, developed during the 1950s, had potential in facilitating mass vaccination campaigns. Adjustments to the 1950's model made this tool possible to administer as many as 1000 vaccinations per hour and not rely on an electrical power source, particularly useful when administering vaccines in remote areas of the world. In 1965, the US government committed to providing assistance to regional smallpox eradication programs in Africa. The USSR, along with other WHO delegations, proposed a program and budget for an intensified program. This intensified program was approved in 1966, with preparations to begin in 1967.

The intensified program contained many components that are used in eradication campaigns today. These include among others, research, surveillance-containment, and routine reporting of cases. By 1970, there was optimism that smallpox could be fully eradicated, with only 18 countries reporting endemic cases that year. However, in 1971, for the first time since the eradication program began, an increase in endemic smallpox was reported. Civil war, unsatisfactory local eradication programs, and new cases in countries previously thought to be free of the epidemic contributed to the rising number of cases. An even more intensified program, "Target Zero", was created with a goal of concentrating on the few remaining endemic countries to eradicate smallpox by June 1974. By 1976, endemic smallpox was only known to exist in 66 villages in Ethiopia. Finally, on May 8, 1980, the World Health Assembly declared successful eradication of smallpox.

\section{Global Collaborations}

Reminiscent of the declaration by the $\mathrm{WHO}$ on eradicating smallpox, in 2000, the United Nations created the Millennium Declaration stating within its goals to "Have halted by 2015 and begun to reverse the spread of HIV/AIDS." HIV/AIDS was then recognized to not only be an individual health issue, but also one that also created economic instability within a nation. Disproportionally affecting women and girls, creating numerous orphans, and deaths of workers in their prime would cause countries to remain in poverty. This was followed in 2001 with the UN Declaration of Commitment in HIV/ AIDS, a commitment by governments globally to combat HIV/AIDS

${ }^{*}$ Corresponding author: Murray R. Berkowitz, Preventive and Community-Based Medicine, Philadelphia College of Osteopathic Medicine - Georgia Campus, 625 Old Peachtree Road NW, Suwanee, Georgia 30024, USA, Tel: 678-225-7482, 202256-2023; E-mail: murraybe@pcom.edu

Received May 30, 2013; Accepted July 25, 2013; Published July 31, 2013

Citation: Chung A, Berkowitz MR (2013) Comparison of Smallpox vs. HIVIAIDS Eradication Strategies. J AIDS Clinic Res S6: 003. doi:10.4172/2155-6113.S6-003

Copyright: ( 2013 Chung A, et al. This is an open-access article distributed under the terms of the Creative Commons Attribution License, which permits unrestricted use, distribution, and reproduction in any medium, provided the original author and source are credit ed. 
in alignment with the Millennium Development Goals. In 2003, the United States President's Emergency Plan for AIDS Relief (PEPFAR), a US based initiative for world AIDS relief, was created [3]. Historically, it is the largest effort by any nation geared towards combating a single disease [4].

Much like the evolution of the original smallpox eradication program, the global HIV/AIDS strategy has also sharpened its focus on more efficient efforts and common goals. In 2011, the United Nations strategy for "Getting to Zero" set a goal of "Zero new HIV infections, Zero discrimination, and Zero AIDS-related deaths." This new strategy lays out a goal of achieving universal access to HIV prevention, treatment, care and support, also in alignment with the Millennium Development Goals. That same year, both the WHO and the US Government established strategic plans in line with "Getting to Zero" campaign. The WHO global health sector strategy on HIV/AIDS, 2011-2015 outlines the WHO's strategy for two main goals: universal access to HIV prevention, diagnosis, treatment and care interventions and to contribute to achieving the UN Millennium Development Goals by 2015 [5]. The US Government committed to expanding its goals into creating an AIDS-free generation through PEPFAR. An AIDS-free generation is being defined as one where" virtually no children are born with HIV and individuals live successful with HIV due to early, sustained treatment." This new strategy emphasized expanding Anti-Retroviral Treatment (ART), Prevention of Mother to Child Transmission (PMTCT), voluntary medical male circumcision, increased HIV counseling and testing services, and continued support for correct and consistent condom use [6]. By reducing new infections and increasing the number of patients on ART, the risk of HIV/AIDS infection should be significantly lowered.

\section{Vaccine Challenges}

While eradication of smallpox proved to be a global success, HIV/AIDS has proven to be much more elusive. Key to the success of eradicating smallpox was the development of a vaccine. Unfortunately yet another HIV vaccine hopeful, HVTN 505, failed this year, with the study being halted by the National Institutes of Health (NIH). This particular study focused on populations of Men Who Have Sex with Men (MSM) and transgendered individuals who have sex with men, both high-risk populations for HIV/AIDS in the US. Preliminary results of the study showed that neither prevention of the disease nor a decrease in viral load occurred in recipients of the vaccine [7]. Thus, the study was halted.

Despite the latest failed vaccine study, there is still hope for a vaccine in the horizon. In March 2013, a case report was released of a 2-year from Mississippi who is reportedly "functionally cured" of HIV. Aggressive treatment was administered within 30 hours of the child's birth. The mother of the child had not received a positive diagnosis of HIV until just prior to the birth, and subsequently had not received any prenatal HIV treatment. The child received therapy for about 15 months before the mother stopped treatment. At this time, viral load has remained undetected in the child [8]. Later in that same month, French scientists released a study recognizing 14 people who might also be "functionally cured" [9]. These subjects began HIV treatment early in their primary infection. Viral load in these subjects were so low, they are "functional cured," as in the virus is still within them, but does not require any treatment for control. Both reports highlight the need to further study the effects of early intervention treatment with primary infection of HIV. With the case of the Mississippi child, the fact that the mother did not receive prenatal HIV treatment may have implications in developing countries where prenatal HIV treatment is not yet the standard.

\section{HIV/AIDS Detection}

In addition, unlike smallpox, HIV infection is not readily seen by the naked eye. A characteristic rash that could be identified by both program staff and villagers could detect presence of smallpox. Identification of this rash was reported to that nation's smallpox surveillance system, which became essential to targeting populations for the eradication campaigns. HIV/AIDS, on the other hand, relies on laboratory data for estimating both prevalence and incidence in a population. This requires the healthcare worker to properly prepare, store, and transport the specimens to a laboratory facility. Accuracy of the data also relies on the strength of that particular country's casereporting system [5].

\section{Prevention and treatment}

In 2008, an estimated 430,000 children were newly infected with HIV, mostly through Mother-To-Child Transmission (MTCT). Globally, HIV/AIDS is now the leading cause of mortality among women of reproductive age [10]. Current Antiretroviral Treatment (ART) eligibility is based on CD4 cell count or WHO clinical stages. Earlier this year, the CDC reported on an important innovation from the Malawi Minister of Health. The strategy, called Option B+, called for all HIV-infected pregnant and breastfeeding women to be eligible for lifelong ART, regardless of CD4 count. This eliminates the extra step of obtaining a CD4 count from laboratories, which are limited in certain areas of the world [11]. Moreover, the WHO recently stated that the Option $\mathrm{B}+$ policy, although requiring more financial resource initially, is a cost-effective measure, in the long-run [10]. Option B+ could be a catalyst for eliminating mother-to-child transmission of HIV worldwide, a goal in the "Getting to Zero" campaign.

In addition to MTCT, it has also been shown that Voluntary Medical Male Circumcision (VMMC) can reduce HIV infection by about $60 \%$. VMMC has the added benefit of also reducing risk of infection of other diseases, such as HPV, cervical cancer, and other sexually transmitted infections. Unlike ART, which requires chronic therapy, VMMC is a one-time procedure with benefits lasting a lifetime. VMMC's benefits are most effective in populations with HIV infections that are primarily heterosexually-driven, and also have a low rate of male circumcision [12-14]. Those undergoing VMMC are also tested, counseled, and educated on HIV infection. Political and religious commitment was shown to increase the rate of VMMC and a decrease in HIV infection $[15,16]$.

Technology is being used to help identify where to concentrate scarce resources. Xia and Moog applied nonlinear mathematical and systems modeling to developing HIV/AIDS models. They concluded the 4-dimensional model developed would be useful in formulating guidelines for clinical practice [17]. Geomapping has been used to indentify population surveillance data geographically to help the preventive medicine and public/community health resources to be better applied where needed [18]. Hay and associates found over 20 maps of HIV/AIDS in the published literature but found that "less than 5 per cent of clinically important infectious diseases have been mapped reliably" [19].

\section{Conclusion}

This year marks the $30^{\text {th }}$ anniversary of the discovery of HIV. Since then, much progress has been made to stymie the progression of the disease. HIV/AIDS has gone from a death sentence to a treatable disease. Aligning and collaborating global strategies and goal has been instrumental in producing more efficient policies and positive 
Citation: Chung A, Berkowitz MR (2013) Comparison of Smallpox vs. HIVIAIDS Eradication Strategies. J AIDS Clinic Res S6: 003. doi:10.4172/2155-6113. S6-003

results. Sharing of information among countries, such as Option B+, has helped efficiently target high-risk populations with better policies. Although many strategies are in place, this article strives to highlight those strategies that align globally under the UN "Getting to Zero" campaign. Until a vaccine or cure exists, current global strategy efforts should continue to focus on prevention and treatment of the disease. As pointed out by Fenner and associates, the costs associated with preventive medicine efforts need to be both inexpensive and simple to apply and implement [2]. The potential of geo mapping HIV/AIDS will required the availability of a large amount of systematically collected prevalence data but would be the first model-based geostatistics application to STDs such as HIV [19].

Historically, the smallpox eradication program took longer than anticipated, with the eradication program constantly evolving to target endemic countries while strengthening the global surveillance and reporting system. However, in the end, the smallpox eradication programs have shown the world that eradication of a disease is possible. Given the lack of an effective HIV vaccine, prevention strategies will require greater political commitment than has been shown, and will most likely require more resources. The hope is that HIV/AIDS can also be eradicated.

\section{References}

1. UNAIDS (2012) World AIDS Day Report.

2. Fenner F, Henderon DA, Arita I, Jezek Z, Ladnyi ID (1988) Smallpox and Its Eradication. World Health Organization, Geneva.

3. Salaam-Blyther T (2012) The President's Emergency Plan for AIDS Relief (PEPFAR): Funding Issues after a Decade of Implementation, FY 2004 FY2013. Congressional Research Service, Washington, DC.

4. Kates J, Wexler A, Lief E, Golub B (2012) Financing the Response to AIDS in Low- and Middle-Income Countries: International Assistance from Donor Governments in 2011. Kaiser Family Foundation and UNAIDS.

5. WHO (2011) When and how to use assays for recent infection to estimate HIV incidence at a population level. Global Health Sector Strategy on HIVIAIDS 2011-2015.
6. CDC (2012) CDC Global Health Strategy, 2012-2015.

7. AIDS Research Alliances (2013) NIH Discontinues Immunizations in HIV Vaccine Study.

8. Persaud D, Gay H, Ziemnaik C, Chen YH, Piatak M, et al. (2013) Functional HIV Cure after Very Early ART of an Infected Infant. 20th Conference on Retroviruses and Opportunistic Infections.

9. Sáez-Cirión A, Bacchus C, Hocqueloux L, Avettand-Fenoel V, Girault I, et al. (2013) Post-Treatment HIV-1 Controllers with a Long-Term Virological Remission after the Interruption of Early Initiated Antiretroviral Therapy ANRS VISCONTI Study. PLoS Pathog 9: e1003211.

10. WHO (2010) Preventing mother-to-child transmission of HIV to reach the UNGASS and Millennium Development Goals. PMTCT Strategic Vision 2010 2015.

11. Centers for Disease Control and Prevention (CDC) (2013) Impact of an innovative approach to prevent mother-to-child transmission of HIV--Malawi, July 2011-September 2012. MMWR Morb Mortal Wkly Rep 62: 148-151.

12. Szabo R, Short RV (2000) How does male circumcision protect against HIV infection? BMJ 320: 1592-1594.

13. Clark S (2000) Male circumcision could help protect against HIV infection. Lancet 356: 225.

14. Weiss HA (2007) Male circumcision as a protective measure against HIV and other sexually transmitted diseases. Curr Opin Infect Dis 20: 66-72.

15. Short RV (2006) New ways of preventing HIV infection: thinking simply, simply thinking. Philos Trans R Soc Lond B Biol Sci 361: 811-820.

16. Tarimo EA, Francis JM, Kakoko D, Munseri P, Bakari M, et al. (2012) The perceptions on male circumcision as a preventive measure against HIV infection and considerations in scaling up of the services: a qualitative study among police officers in Dar es Salaam, Tanzania. BMC Public Health 12: 529

17. Xia X, Moog CH (2003) Indentifiability of Nonlinear Systems with Application to HIVIAIDS Models. IEEE Transactions on Automatic Control 48: 330-336.

18. National Alliance of State and Territorial AIDS Directors (2007) HIV Prevention Targeting Populations Using Geo-Mapping and Social Network Strategies.

19. Hay SI, Battle KE, Pigott DM, Smith DL, Moyes CL, et al. (2013) Global mapping of infectious disease. Philos Trans R Soc Lond B Biol Sci 368: 20120250. 